\title{
Die Gelegenheit nutzen: Die Evaluation der Friedens- und Konfliktforschung durch den Wissenschaftsrat aus der Perspektive der Arbeitsgemeinschaft für Friedens- und Konfliktforschung (AFK)
}

\author{
Bettina Engels $\cdot$ Conrad Schetter
}

Online publiziert: 20. April 2020

(C) Der/die Autor(en) 2020

Zusammenfassung In diesem Forumsbeitrag kommentieren wir die aktuelle Evaluation der Friedens- und Konfliktforschung in Deutschland durch den Wissenschaftsrat als (ehemalige) Vorsitzende der Arbeitsgemeinschaft für Friedens- und Konfliktforschung (AFK e. V.). Wir reflektieren, was die Evaluation aus der Perspektive der AFK für das Forschungsfeld der Friedens- und Konfliktforschung und für den Verband selbst bedeutet. Dazu stellen wir dar, wie die AFK als Fachverband in der Evaluation berücksichtigt wurde. Anschließend beleuchten wir zentrale Themenfelder, die im Evaluationsbericht angesprochen werden: Professionalisierung, die normative Ausrichtung des Forschungsfelds, Interdisziplinarität, Internationalisierung, die Standortfrage, politischer Nutzen, Finanzierung und Nachwuchsförderung. Wir argumentieren, dass die Evaluation eine Gelegenheit für das Forschungsfeld generell und für die AFK im Besonderen darstellt, um über die Stärken und Perspektiven der Friedens- und Konfliktforschung in Deutschland reflektieren und Strategien entwickeln, diese strukturell und institutionell zu sichern und auszubauen. Die AFK als standort- und institutionenübergreifender Fachverband könnte hierbei und als Lobbyorganisation eine wichtige Rolle übernehmen.

Schlüsselwörter Vernetzung · Internationalisierung · Interdisziplinarität · Professionalisierung $\cdot$ Nachwuchsförderung

\footnotetext{
B. Engels $(\bowtie)$

Nachwuchsgruppe GLOCON, Freie Universität Berlin, Boltzmannstr. 1, 14195 Berlin, Deutschland E-Mail: bettina.engels@fu-berlin.de

C. Schetter

bicc/Internationales Konversionszentrum Bonn, Pfarrer-Byns-Straße 1, 53121 Bonn, Deutschland

E-Mail: schetter@bicc.de
} 


\title{
Taking the opportunity: The evaluation of peace and conflict studies in Germany from the perspective of the German Association for Peace and Conflict Studies (Arbeitsgemeinschaft für Friedens- und Konfliktforschung e.V. - AFK)
}

\begin{abstract}
We comment on the German Science Council's recent evaluation of peace and conflict studies from our perspective as (previous) chairpersons of the German Association for Peace and Conflict Studies (Arbeitsgemeinschaft für Friedens- und Konfliktforschung e.V. - AFK). We reflect on the consequences of the evaluation for peace and conflict studies in Germany in general, and the association in particular. Inititially, we present how the AFK is taken into account in the evaluation. Subsequently, we outline main topics that are addressed in the evaluation report: professionalising, normative orientation of research, interdisciplinarity, institutional locations, political utility, funding, and promotion of young scholars. We argue that the evaluation presents an opportunity to reflect on the strengths and perspectives of peace and conflict studies in Germany, and on strategies to institutionally secure and expand them. Thereby, the AFK as a professional association across institutions and locations, could play a major role, notably with respect to lobbying.
\end{abstract}

Keywords Networking · Internationalisation · Interdisciplinarity ·

Professionalising · Young scholars

Die Friedens- und Konfliktforschung geht - 35 Jahre nach der letzten umfassenden Beschäftigung des Wissenschaftsrats mit dem Forschungsfeld - gestärkt aus der jüngsten Evaluation hervor. Schon im Vorfeld und während der Durchführung setzte die Evaluation einen umfassenden Diskussions- und Reflexionsprozess innerhalb der wissenschaftlichen Community über das Selbstverständnis, die inhaltlichen und institutionellen Gegebenheiten und Perspektiven der Friedens- und Konfliktforschung in Gang. Die Arbeitsgemeinschaft für Friedens- und Konfliktforschung e. V. (AFK) als der Fachverband der Friedens- und Konfliktforschung im deutschsprachigen Raum beteiligte sich an diesem Diskussionsprozess proaktiv. Der Verband als solcher wurde durch den Wissenschaftsrat im Zuge der Evaluierung zwar nicht zu einer Stellungnahme aufgefordert. Dennoch organisierte die AFK zusammen mit der Deutschen Stiftung Friedensforschung (DSF) zwei eintägige Treffen zur Positionierung der Friedens- und Konfliktforschung gegenüber der Evaluation, an denen jeweils 15-20 Vertreter*innen der Zentren der deutschen Friedens- und Konfliktforschung teilnahmen: am 26. September 2017 am Bonn International Center for Conversion (BICC) in Bonn und am 29. Januar 2018 an der Goethe-Universität in Frankfurt/Main.

Von Beginn des Prozesses an wurden die Evaluation und die mit ihr verbundenen Chancen und Risiken für das Forschungsfeld innerhalb der AFK intensiv diskutiert. Zentrale Aspekte waren dabei die Perspektiven für den wissenschaftlichen Nachwuchs, die Stärkung der Lehre, das Verhältnis unterschiedlicher Akteure im Forschungsfeld (der ,großen “, meist außeruniversitären Forschungsinstitute, der universitären Zentren, Lehrstühle und Studiengänge sowie praxisorientierter For- 
scher*innen und Institutionen), die Verbesserung der Forschungsförderung sowie die Rolle der DSF. Mit der Veröffentlichung der Evaluierung im Juli 2019 erstreckte sich die Diskussion auch in mediale und parlamentarische Arenen (bspw. BT DS 19/14111 2019; taz 2019; vgl. auch Gehring und Rubner 2020). Auch im politischen Raum findet die AFK als Standesvertretung Gehör. So wurde die AFK zur Anhörung zum Antrag von Bündnis 90/Die Grünen „Friedens- und Konfliktforschung stärken“ in den Wissenschaftsausschuss des Landes Nordrhein-Westfalen am 25. März 2020 in den Landtag nach Düsseldorf eingeladen.

Wir kommentieren im Folgenden die Evaluation als (ehemalige) Vorsitzende der AFK in den Jahren 2015 bis 2018 bzw. 2020. Es handelt sich dabei nicht um eine Stellungnahme des Verbands, der einen Konsens der AFK oder ihres Vorstands wiedergibt, sondern um unsere individuelle Reflexion einzelner Aspekte, von denen wir glauben, dass sie für die AFK relevant sind. Wir möchten darstellen, was die aktuelle Evaluation durch den Wissenschaftsrat aus der Perspektive der AFK für das Forschungsfeld der Friedens- und Konfliktforschung und für den Verband selbst bedeutet. In einem ersten Schritt würdigen wir kritisch, wie die AFK als Fachverband im Evaluationsbericht berücksichtigt wurde. In einem zweiten Schritt beleuchten wir zentrale Themenfelder, die im Evaluationsbericht angesprochen werden und die wir für die AFK für wichtig halten: Professionalisierung, die normative Ausrichtung des Forschungsfelds, Interdisziplinarität, Internationalisierung, die Standortfrage, politischer Nutzen, Finanzierung und Nachwuchsförderung. Wir diskutieren, wie die AFK sich in diesen Themen in Zukunft einbringen könnte. Insgesamt bietet die Evaluation durch den Wissenschaftsrat eine hervorragende Gelegenheit für das Forschungsfeld generell und für die AFK im Besonderen - so unser Resümee: Die ForschungsCommunity sollte den begonnenen Diskussionsprozess fortsetzen, über die Stärken und Perspektiven der Friedens- und Konfliktforschung reflektieren und Strategien entwickeln, diese strukturell und institutionell zu sichern und auszubauen. Der Evaluationsbericht könnte ein Gelegenheitsfenster für die politische Lobbyarbeit auf der Ebene des Bundes und der Länder zugunsten der Friedens- und Konfliktforschung darstellen. Die AFK als standort- und institutionenübergreifender Fachverband kann und sollte hierbei eine wichtige Rolle übernehmen.

\section{Die AFK als Gegenstand der Evaluation}

Die AFK als ,wissenschaftliche Fachgesellschaft der Friedens- und Konfliktforschung" (Wissenschaftsrat 2019, S. 24) findet im Evaluationsbericht nur an sehr wenigen Stellen Erwähnung; dann aber stets positiv. In der Evaluation finden sich keine konkreten Empfehlungen, die eine Neuausrichtung oder eine Kursänderung der AFK bedingen würden. Der Bericht fasst die zentralen Aktivitäten der AFK und ihre Bedeutung für die Friedens- und Konfliktforschung folgendermaßen zusammen:

Zur überregionalen Vernetzung der deutschen Friedens- und Konfliktforschung trägt die Arbeitsgemeinschaft für Friedens- und Konfliktforschung (AFK) mit ihren zehn Arbeitskreisen, einer jährlichen Tagung und der von ihr herausgegebenen Zeitschrift für Friedens- und Konfliktforschung (ZeFKo) bei (Wissenschaftsrat 2019, S. 91). 
Als eine Stärke der AFK hebt die Evaluation die überregionale Netzwerkbildung hervor, die rundum positiv bewertet wird. Die Evaluation würdigt vor allem die aktive Rolle der Arbeitskreise, etwa des AK Curriculum und Didaktik:

Der intensive Austausch zwischen den Lehrenden im Arbeitskreis Curriculum und Didaktik der Arbeitsgemeinschaft für Friedens- und Konfliktforschung ist von einer bemerkenswerten kritischen Selbstreflexion getragen und befördert gleichermaßen die Weiterentwicklung didaktischer Konzepte wie die standortübergreifende Zusammenarbeit, etwa in internetgestützten Ringvorlesungen. Der Wissenschaftsrat bestärkt die Lehrenden der Friedens- und Konfliktforschung darin, an dieser Zusammenarbeit festzuhalten. Diese ist für kleinere Standorte und für Wissenschaftlerinnen und Wissenschaftler in der Qualifizierungsphase besonders wichtig (Wissenschaftsrat 2019, S. 43).

Über die wiederkehrenden Workshops, die Teilnehmer*innen unterschiedlicher Universitäten, Fachhochschulen und außeruniversitärer Institutionen und Organisationen zusammenbringen, schafft der AK Curriculum und Didaktik Austausch und Synergien zwischen den Studiengängen und unterstützt deren Profilbildung. Durch die mit den Workshops verbundenen Trainings trägt der AK wesentlich zur didaktischen Qualifizierung der Lehrenden im Bereich der Friedens- und Konfliktforschung und damit zur Qualität der Lehrangebote bei. Die Evaluierung war Anlass für den AK Curriculum und Didaktik, sich im November 2017 über das Selbstverständnis des AK und der Studiengänge zu verständigen. Die dazu veröffentlichte „Augsburger Erklärung“ (ohne Jahr) des AK wird im Evaluationsbericht explizit erwähnt.

Außerdem unterstreicht der Bericht die Vernetzung junger Wissenschaftler*innen im AK Junge AFK:

Positiv zu bewerten sind die Tagungen der „Jungen AFK“ der Arbeitsgemeinschaft für Friedens- und Konfliktforschung, die einerseits dem Austausch und der Vernetzung der jüngeren Friedens- und Konfliktforscherinnen und -forscher untereinander und andererseits der Diskussion ihrer Papiere mit etablierten Wissenschaftlerinnen und Wissenschaftlern dienen (Wissenschaftsrat 2019, S. 45).

Insgesamt sieht der Wissenschaftsrat den Beitrag der AFK zur Friedens- und Konfliktforschung in erster Linie in ihren vielfältigen Vernetzungsaktivitäten. Dabei hebt der Bericht vor allem die Arbeit der AKs hervor. Weniger Aufmerksamkeit widmet er anderen Aktivitäten des Verbands, die gleichermaßen wichtig für die Netzwerkarbeit der AFK sind: das jährliche Kolloquium, die ZeFKo, die Homepage und der Newsletter.

\section{Zentrale Themen und ihre Relevanz für die AFK}

\subsection{Professionalisierung}

Die (akademische) Professionalisierung der Friedens- und Konfliktforschung zieht sich wie ein roter Faden durch den Evaluationsbericht und wird dabei insgesamt als 
positiv erachtet. Sie drückt sich in der Schaffung und Besetzung von Professuren, der Gestaltung von Studiengängen, Peer-review-Verfahren u.ä. aus. Die AFK ist an diesem Prozess aktiv beteiligt: mit der Einrichtung einer professionellen, mit einer hauptamtlichen Kraft besetzten und an einer Hochschule angesiedelten Geschäftsstelle seit 2010; mit der Umstellung des Formats der Kolloquien auf einen offenen Call for Panels and Papers unter einem weit gefassten und aktuellen Oberthema seit 2011; mit der Gründung der ZeFKo 2012 als begutachteter Zeitschrift, die internationalen Standards entspricht und mittlerweile einen relevanten Anteil englischsprachiger Beiträge enthält. Wir schätzen diese Entwicklung der AFK in den letzten zehn Jahren als positiv und weitgehend gelungen ein: Die Friedens- und Konfliktforschung braucht einen aktiven und professionell organisierten Fachverband, der Vernetzung ermöglicht, Diskussionsräume schafft, kritische Auseinandersetzungen und Reflexion nach innen fördert und das Feld nach außen gegenüber gesellschaftlichen und politischen Akteuren und Medien vertritt. Die Professionalisierung des Forschungsfelds bedeutete allerdings auch, dass neue Herausforderungen auftreten, in denen die AFK eine wichtige Rolle spielen kann. Ein zentrales Feld ist hier die Schnittstelle zwischen Wissenschaft einerseits und politischer Praxis, Zivilgesellschaft und sozialen Bewegungen andererseits. Die Vielfalt der in dem Feld beteiligten Akteure und ihrer Perspektiven ist für die AFK heute so wichtig wie zu Zeiten ihrer Gründung 1968. Dies schlägt sich in entsprechenden Workshops, Panels und Podiumsdiskussion im Rahmen der AFK-Kolloquien nieder. Bereits seit einigen Jahren existiert ein aktiver Arbeitskreis Wissenschaft und Praxis. Auch die Beteiligung der AFK an der Plattform Zivile Konfliktbearbeitung sowie der Redaktion und im Vorstand der Zeitschrift Wissenschaft und Frieden $(\mathrm{W}+\mathrm{F})$ sind institutionalisierte Formen des engen Austausches von Wissenschaft und politischer Praxis im Rahmen der Aktivitäten des Verbands. Die AFK könnte die positive Einschätzung der zunehmenden Professionalisierung durch den Evaluationsbericht zum Anlass nehmen, das Verhältnis der wissenschaftlichen Friedens- und Konfliktforschung im allgemeinen und der AFK im Besonderen dezidiert zum Gegenstand einer kritischen Reflexion im Rahmen eines Workshops oder einer Plenumsdiskussion bei einem zukünftigen Kolloquium zu machen.

\subsection{Normative Ausrichtung des Forschungsfelds}

Der Evaluationsbericht stellt fest, dass mit der Professionalisierung die Grenzen der Friedens- und Konfliktforschung gegenüber der sicherheitspolitischen Forschung aufgeweicht worden seien, denn auch für die Friedens- und Konfliktforschung bildet Sicherheit einen wichtigen Forschungsgegenstand. Jedoch kommt eine zentrale Stärke der Friedens- und Konfliktforschung, die sie vom Mainstream der Sicherheitsforschung unterscheidet, in dem Gutachten zu kurz: dass sie über die normativen Grundlagen ihrer Forschung reflektiert und diese offenlegt. Das spiegelt sich gegenwärtig weniger in grundlegenden Kontroversen und ,Grabenkämpfen ‘ wider, sondern vielmehr in tiefgehenden kritischen Auseinandersetzungen über Theorien, Begriffe und Methoden und deren gesellschaftlich-politische Implikationen (siehe auch Niemann und Schröder 2020). Dies ist bei den jährlichen Kolloquien der AFK, den Tagungen der Jungen AFK und den Workshops der AKs deutlich sichtbar: Dort wid- 
men sich regelmäßig Beiträge und Panels den Fragen der normativen Grundlagen der Forschung. Dabei wird sichtbar, dass feministische und postkoloniale Ansätze im deutschsprachigen Raum in der Friedens- und Konfliktforschung inzwischen weitgehend akzeptiert und relativ weit entwickelt sind.

Hier zeigt sich zudem, dass gerade die Friedens- und Konfliktforschung und die kritische Sicherheitsforschung über ähnlich gelagerte Reflexionsansätze miteinander verbunden sind. Die AFK könnte die Frage nach der Abgrenzung der Friedensund Konfliktforschung gegenüber dem Mainstream der Sicherheitsforschung auf der einen Seite sowie Schnittstellen mit der kritischen Sicherheitsforschung auf der anderen Seite aktiv aufgreifen, beispielsweise auf einem der kommenden Kolloquien, in einer ZeFKo-Debatte oder durch ein gemeinsames Panel oder einen Workshop in Zusammenarbeit mit der DVPW-Themengruppe Kritische Sicherheitsforschung. Die Diskussion der Unterschiede und Schnittstellen zwischen den Zugängen beider Forschungsfelder könnte eine Gelegenheit bieten, die über Jahrzehnte etablierte normative Reflexion als eine wesentliche Grundlage der Friedens- und Konfliktforschung und als ein wissenschaftliches Aushängeschild zu stärken.

\subsection{Interdisziplinarität}

Die Friedens- und Konfliktforschung stellt ein Forschungsfeld dar - keine Disziplin. Darauf weist auch der Evaluationsbericht hin. Aus dem Gutachten wird aber auch ersichtlich, dass die Professionalisierung der Friedens- und Konfliktforschung zu einem Verlust an Interdisziplinarität geführt hat. Gegenwärtig scheint das Forschungsfeld in Deutschland von der Politikwissenschaft und den Internationalen Beziehungen dominiert. Der Evaluationsbericht stellt fest, dass die naturwissenschaftliche Friedens- und Konfliktforschung, die einst ein internationales Flaggschiff der deutschen Forschung darstellte, weitgehend weggebrochen ist (vgl. den Beitrag von Reuter et al. 2020). Das spiegelt sich auch in der AFK wider: Die Technik-, Natur- und Lebenswissenschaften sind im Verband vergleichsweise gering vertreten, und auch auf den Kolloquien und in der ZeFKo finden sich gegenwärtig sehr wenige Beiträge aus diesen Feldern. Entsprechend sollte die AFK stärker gezielt auf Kolleg*innen aus dem Bereich der naturwissenschaftlichen Friedens- und Konfliktforschung zugehen, um sie in die Vernetzungsaktivitäten einzubinden, etwa über den „Forschungsverbund Naturwissenschaft, Abrüstung und internationale Sicherheit" (FONAS). ${ }^{1}$ Dass Vertreter der FONAS sich aktiv in den mit der Evaluation verbundenen Diskussionsprozess einbringen, stellt ein Chance dar, welche die AFK nutzen sollte, um durch den persönlichen Kontakt die beiden Netzwerke enger miteinander zu verflechten. Damit meinen wir nicht die Gründung eines AK Naturwissenschaftliche Friedensund Konfliktforschung oder ähnliches parallel zur FONAS, sondern mögliche gemeinsame Aktivitäten von AFK und FONAS. Darüber hinaus ist es wünschenswert, mehr Natur- und Technikwissenschaftler*innen als aktive Mitglieder für die AFK zu gewinnen.

\footnotetext{
1 In FONAS sind Wissenschaftler*innen interdisziplinärer Forschungsgruppen verschiedener Universitäten (u. a. RWTH Aachen, TU Darmstadt, TU Dortmund, des Forschungszentrums Jülich, der HSFK, des IFSH) vernetzt, die zu Fragen des Zusammenhangs von Technologie und Frieden arbeiten.
} 
Allerdings gerät in der Evaluation aus dem Blick, dass sich Inter- und Transdisziplinarität keineswegs auf die Zusammenarbeit von Geistes- und Sozialwissenschaften auf der einen und Natur- und Technikwissenschaften auf der anderen Seite beschränkt. Das Spektrum sozial- und geisteswissenschaftlicher Fachdisziplinen, die im Forschungsfeld der Friedens- und Konfliktforschung - und entsprechend auch in der AFK - vertreten sind, sein könnten oder sein sollten, ist breit: Geographie, Anthropologie, Ökonomie, Religionswissenschaften, Kultur- und Medienwissenschaften, Pädagogik, Geschichtswissenschaft, Literaturwissenschaften und andere. Eine tiefere Verankerung der Friedens- und Konfliktforschung in der Breite der Geistes- und Sozialwissenschaften könnte das Forschungsfeld institutionell stärken und inhaltlich bereichern. Um dies zu erreichen, kann die AFK als Fachverband eine federführende Rolle übernehmen. Eine zentrale Maßnahme hierfür ist es, entsprechende Kolleg*innen zu identifizieren und gezielt für die aktive Beteiligung im Verband - insbesondere auch im Vorstand - und an seinen Aktivitäten zu gewinnen.

\subsection{Internationalisierung}

Als eine Schwäche der deutschen Friedens- und Konfliktforschung identifiziert die Evaluation, dass sie international noch nicht hinreichend sichtbar sei. Für die AFK ist Internationalisierung schon seit einigen Jahren ein zentrales Thema. Der Fachverband hat wichtige Schritte unternommen, um die internationale Vernetzung und Sichtbarkeit deutschsprachiger Friedens- und Konfliktforscher*innen zu verbessern: Das Jahreskolloquium 2017 fand in Kooperation mit der European Peace Research Association (EuPRA) und erstmalig vollständig englischsprachig statt. Auch bei den folgenden Kolloquien fand ein zunehmender Teil der Panels in englischer Sprache statt. Für die Keynote-Vorträge bei den Kolloquien konnten bereits mehrfach renommierte internationale Wissenschaftler*innen gewonnen werden (beispielsweise 2015 Tarek Barkawi; 2016 Severine Autessere; 2019 Oliver Richmond; 2020 Amina Mama). Die ZeFKo ermöglicht mittlerweile ebenfalls die Publikation englischsprachiger Beiträge. Ohnehin versteht sich die AFK nicht als deutscher Fachverband, sondern als die Vereinigung der deutschsprachigen Friedens- und Konfliktforscher*innen, in der Kolleg*innen aus Österreich und der Schweiz ebenfalls stark vertreten sind.

\subsection{Standortfrage}

Die Evaluation legt nahe, dass sich drei bis vier konzentrierte Standorte der Friedens- und Konfliktforschung in Deutschland herausbilden sollten. Wissenschaftspolitisch lassen sich Argumente für (Clusterbildung, Themenkonzentration) und gegen (Mainstreaming, Einschränkung der Vielfalt der Themen und Perspektiven) eine solche örtliche Konzentration finden. Insbesondere mit Blick auf das Ziel, Interdisziplinarität zu erhöhen, aber auch hinsichtlich von Wissenschaft-Praxis-Transfer erscheint uns die Konzentration auf wenige, vermeintlich zentrale Standorte jedoch wenig zielführend. Denn die Zusammenarbeit mit Praxispartner*innen findet keinesfalls nur an den offenkundig bundespolitisch relevanten Standorten wie Berlin oder Bonn statt, sondern gerade auch auf kommunaler Ebene. Dies hat die erfolgreiche 
Zusammenarbeit der AFK auf lokaler Ebene beispielsweise mit der Friedensstadt Augsburg und der Stadt Kleve gezeigt. Aus Sicht der AFK als interdisziplinärem Fachverband hat sich die dezentrale Vernetzungsarbeit bewährt, die dazu beiträgt, die Friedens- und Konfliktforschung auch an Standorten zu stärken, die eher peripher zu vermeintlichen Zentren liegen. In diesem Sinne finden die Jahreskolloquien der AFK mit großem Erfolg an wechselnden Standorten (z. B. 2019 Erfurt, 2020 in Schwerte/Westfalen, 2021 in Magdeburg) statt.

\subsection{Politischer Nutzen}

Die Initiative für die Evaluation kam aus dem Bundestag, und das gestiegene Interesse an der Friedens- und Konfliktforschung steht auch im Zusammenhang mit der Frage nach „Deutschlands gestiegener internationaler Verantwortung“. Mit diesem politischen Interesse umzugehen, stellt für die Forschung eine Gratwanderung zwischen unabhängiger Wissenschaft und politischem Beratungsbedarf dar. Besonders problematisch wird dies, wenn Wissenschaftsförderung eng an den vermeintlichen politischen und wirtschaftlichen Nutzen der Forschung geknüpft ist; dies spiegelt sich beispielsweise im BMBF-Programm „Forschung für zivile Sicherheit“ wider. Wir beobachten eine Tendenz, dass im Bereich der Friedens- und Konfliktforschung vor allem Wissen mit vermeintlich hohem, unmittelbar erkennbarem politisch-praktischem und technischem Nutzen als förderungswürdig befunden wird. Demgegenüber ist die Projektfinanzierung für Grundlagenforschung, vor allem zu normativen Fragen, schwieriger zu erreichen. Häufig scheint das Interesse an kleinteiliger, wirkungsorientierter Forschung, zum Beispiel zu Maßnahmen von unterschiedlichen Formen von Interventionen, Mediation, Sicherheitssektorreformen u. ä. größer als am grundlegenden Hinterfragen und Verändern der Rahmenbedingungen von Konflikt, Gewalt und Frieden.

Die AFK kann hier als Fachverband auf zwei Ebenen eine wichtige Rolle einnehmen: Zum einen kann sie - etwa bei den Kolloquien, den Workshops der AKs und in der ZeFKo - Räume bieten, um eben jene grundlegenden und normativen Fragen zu diskutieren. Zum anderen sollte sie sich kritisch in die wissenschaftspolitische Diskussion einbringen, Fragen beispielsweise der Förderpolitik öffentlich thematisieren und politische Lobbyarbeit für eine tatsächlich inter- und transdisziplinäre und grundlagenorientierte Friedens- und Konfliktforschung betreiben.

\subsection{Finanzierung}

Gute Forschung und gute Lehre kosten Geld; das ist unbestritten. Um Grundlagenforschung voranzubringen, über kleinteilige und tagespolitisch aktuelle Aspekte hinauszudenken, zentrale zukunftsweisende Fragen zu identifizieren und zu bearbeiten, sind Strukturen und Institutionen der Forschungsförderung notwendig, die Flexibilität im Format und Umfang der beantragten Vorhaben ermöglichen und themenoffene Förderangebote bereitstellen.

Es ist gleichermaßen unbestritten, dass die Friedens- und Konfliktforschung unterfinanziert ist und weit mehr leisten könnte, wäre sie besser ausgestattet. Im Mittelpunkt der Evaluation stand hinsichtlich der Finanzierung von Friedens- und Konflikt- 
forschung die DSF (siehe Schneckener und Held 2020). Die Evaluation verdeutlicht, dass eine erhebliche Aufstockung des Stammkapitals unbedingt notwendig ist. Diese Forderung wird von der Forschungs-Community in Deutschland einhellig begrüßt und unterstützt. Aber die bundespolitische Unterstützung der Friedens- und Konfliktforschung sollte sich keinesfalls auf die Aufstockung des Stiftungskapitals der DSF beschränken. In diesem Sinne fordert auch die Evaluation eine neue Förderlinie des BMBF, die im Sommer 2020 mit einer Gesamtsumme von 30 Mio. $€$ für fünf Jahren eingerichtet werden soll (Deutscher Bundestag 2020, S. 18350). Dass das BMBF so proaktiv und schnell auf eine Evaluation des Wissenschaftsrats mit der Einrichtung einer eigenen Förderlinie reagiert, spiegelt den hohen politischen Stellenwert der Friedens- und Konfliktforschung wider.

Die Friedens- und Konfliktforschung braucht eine aktive Lobby, um nachhaltig für eine bessere Ausstattung des Forschungsfeldes zu werben. Denkbar und wünschenswert wären langfristig und finanziell attraktive Förderprogramme des BMBF, die über einen einmaligen 5-Jahreszyklus hinausgehen. Solche Programme legte das BMBF etwa für die Regionalstudien oder für die Forschung für Nachhaltigkeit auf. Auch diese sind, wie an der Geschichte der sozial-ökologischen Forschung in Deutschland deutlich wird, erst durch wissenschaftspolitische Lobbyarbeit zustande gekommen. Der Evaluationsbericht bietet ein Gelegenheitsfenster für solche Lobbyarbeit zugunsten der Friedens- und Konfliktforschung. Hier kann die AFK als standort- und institutionenübergreifender Verband eine wichtige Rolle spielen. Denn wenn es keine übergreifenden Akteure gibt, die diese Rolle übernehmen, besteht die Gefahr, dass die einzelnen Akteure, die in der Evaluation genannt werden, sich gegenseitig ausspielen, ohne dass die Friedens- und Konfliktforschung dabei als solche insgesamt gewinnt.

Dabei sollte nicht vergessen werden, dass Wissenschaft in Deutschland überwiegend Ländersache ist, und die Finanzierung von Forschung und Lehre vor allem zwischen den Ländern und den Hochschulen ausgehandelt wird. Auch hier kann die AFK eine relevante Rolle übernehmen - weniger als Lobbyorganisation, denn als Netzwerk. Denn durch die breite Verankerung an zahlreichen Standorten bundesweit besteht innerhalb der AFK ein erhebliches Wissen darüber, wie die Friedensund Konfliktforschung in den Bundesländern aufgestellt ist. Die AFK kann den Raum für die Entwicklung von Strategien und die Vernetzung der Lobbyarbeit auf Länderebene bieten.

Auch Vernetzungs- und Verbandsarbeit kostet Geld - vor allem, wenn sie sich nicht auf Ehrenamt, Überstunden, prekäre und nicht hinreichend vergütete Arbeit stützen soll. Zentrale Vernetzungsaktivitäten der AFK wie die Kolloquien, die Tagungen der Jungen AFK, die Workshops des AK Curriculum und Didaktik und andere werden vielfach durch die DSF gefördert. Darüber hinaus wären die Arbeit des Verbands und der ZeFKo jedoch nicht ohne die jeweils mit einer $50 \%$ TVL 13-Stelle ausgestattete professionelle Geschäftsstelle und Redaktion möglich. Die beiden Stellen werden aus Hochschul- bzw. Berufungsmitteln für begrenzte Zeiträume finanziert. Die AFK ist darauf angewiesen, dass engagierte Kolleg*innen erfolgreich mit ihren jeweiligen Hochschulleitungen verhandeln oder bereit sind, Mittel aus der Personalausstattung ihrer Lehrstühle für die Geschäftsstelle der AFK bzw. die Redaktion der ZeFKo zur Verfügung zu stellen. Der Ausbau bestehender 
und die Schaffung neuer Fördermöglichkeiten, die auch die Finanzierung der vom Wissenschaftsrat als positiv und wichtig hervorgehobenen Vernetzungsaktivitäten ermöglichen, wären ein wichtiger Beitrag dazu, die Arbeit der AFK mittelfristig sicherzustellen.

\subsection{Nachwuchsförderung}

Im Bereich der Lehre und Nachwuchsförderung würdigt die Evaluation explizit die entsprechenden Vernetzungsaktivitäten der AFK. Das Gutachten beurteilt die bestehenden Masterstudiengänge sehr positiv und bedauert, dass demgegenüber gegenwärtig keine Promotionsprogramme und Graduiertenkollegs im Bereich der Friedens- und Konfliktforschung in Deutschland existieren. Denkbar sind hier einerseits längerfristig institutionalisierte Graduiertenschulen und Promotionsprogramme und andererseits temporär als Drittmittelvorhaben über die DFG und die Studienwerke der Stiftungen geförderte Promotionskollegs. Insbesondere Letztere werden in Deutschland zunehmend auch standort- und hochschulübergreifend, teilweise auch bereits mit gezielter Beteiligung der Fachhochschulen, eingerichtet.

Um Initiativen für die Einrichtung solcher Promotionsprogramme und Graduiertenkollegs im Bereich der Friedens- und Konfliktforschung zu entwickeln, bietet die AFK als Netzwerk und auch als eine Art institutionelles Gedächtnis eine hervorragende Plattform. Denn in dem Verband sind nicht nur die aktuellen Erfahrungen aller thematisch spezifischen Studiengänge sowie zahlreicher fachdisziplinärer Promotionsprogramme vertreten, sondern besteht darüber hinaus auch erhebliches Wissen über frühere Bestrebungen und Versuche der Einrichtung von Promotionskollegs u. ä. im Bereich der Friedens- und Konfliktforschung.

\section{Fazit}

Die aktuelle Evaluation des Wissenschaftsrats stärkt die Friedens- und Konfliktforschung in Deutschland. Sie bietet ihr Chancen, ihre bestehenden und bewährten Institutionen wie die DSF und die Studienangebote zu sichern und auszubauen sowie potenziell neue Institutionen und Formate auf den Weg zu bringen, etwa im Bereich der Forschungs- und Nachwuchsförderung. Um die sich hier aktuell bietenden Gelegenheiten zu nutzen, sollte die AFK eine wichtige Rolle spielen: als Lobbyistin in der Bundes- und Landespolitik sowie bei den Institutionen der Forschungsförderung; als Arena, die kontroverse Diskussionen und kritische Reflexion innerhalb der Forschungs-Community ermöglicht; als standortübergreifendes Netzwerk, das Vertreter*innen von Hochschulen und außeruniversitären Organisationen zusammenbringt, um ausgehend von der Diskussion im Anschluss an die Evaluation neue Initiativen zu entwickeln und voranzubringen.

Funding Open Access funding provided by Projekt DEAL.

Open Access Dieser Artikel wird unter der Creative Commons Namensnennung 4.0 International Lizenz veröffentlicht, welche die Nutzung, Vervielfältigung, Bearbeitung, Verbreitung und Wiedergabe in jeglichem Medium und Format erlaubt, sofern Sie den/die ursprünglichen Autor(en) und die Quelle ord- 
nungsgemäß nennen, einen Link zur Creative Commons Lizenz beifügen und angeben, ob Änderungen vorgenommen wurden.

Die in diesem Artikel enthaltenen Bilder und sonstiges Drittmaterial unterliegen ebenfalls der genannten Creative Commons Lizenz, sofern sich aus der Abbildungslegende nichts anderes ergibt. Sofern das betreffende Material nicht unter der genannten Creative Commons Lizenz steht und die betreffende Handlung nicht nach gesetzlichen Vorschriften erlaubt ist, ist für die oben aufgeführten Weiterverwendungen des Materials die Einwilligung des jeweiligen Rechteinhabers einzuholen.

Weitere Details zur Lizenz entnehmen Sie bitte der Lizenzinformation auf http://creativecommons.org/ licenses/by/4.0/deed.de.

\section{Literatur}

Augsburger Erklärung. o.J. https://afk-web.de/cms/wp-content/uploads/2018/05/2018_04_Augsburger_ Erklaerung_AFK.pdf.

BT (Hrsg.). 2019. BT DS 19/14111. Antrag der Abgeordneten Kai Gehring u.a. und der Fraktion BÜNDNIS 90/DIE GRÜNEN: Ein Kompass in unruhigen Zeiten - Friedensforschung als Grundlage der Politik stärken. Bd. 19. Berlin: Deutscher Bundestag. 19. Wahlperiode 16.10.2019.

Deutscher Bundestag. 2020. Stenografischer Bericht. 146. Sitzung. Berlin, Donnerstag, den 13. Februar. Tagesordnungspunkt 19: Ein Kompass in unruhigen Zeiten - Friedens-forschung als Grundlage der Politik stärken Drucksache 19/14111, S. 18349-18356. http://dipbt.bundestag.de/dip21/btp/19/ 19146.pdf\#P.18349. Zugegriffen: 10. März 2020.

Gehring, Kai, Henrik Rubner. 2020. Ein wichtiger Kompass in unruhigen Zeiten. Friedensforschung und die Empfehlungen des Wissenschaftsrates aus Perspektive der Politik. Z Friedens und Konffforsch. https://doi.org/10.1007/s42597-020-00031-3.

Niemann, Holger, Ursula Schröder. 2020. Ein Feld in Bewegung: Friedens- und Konfliktforschung in herausfordernden Zeiten. Z Friedens und Konffforsch. https://doi.org/10.1007/s42597-020-00033-1.

Reuter, Christian. 2020. Zur naturwissenschaftlich-technischen Friedens- und Konfliktforschung: Aktuelle Herausforderungen und Bewertung der Empfehlungen des Wissenschaftsrat. Z Friedens und Konflforsch. https://doi.org/10.1007/s42597-020-00035-z.

Schneckener, Ulrich, Thomas Held. 2020. Impulsgeberin und Anstifterin: Die Deutsche Stiftung Friedensforschung im Lichte der Evaluation. Z Friedens und Konfforsch. https://doi.org/10.1007/s42597-02000029-x.

taz. 2019. Wissenschaftsrat über Friedensforschung: „Da steckt viel Beratung drin“. Friedens- und Konfliktforschung muss gestärkt werden, findet der Wissenschaftsrat. Silviana Galassi erklärt, warum das Forschungsfeld wichtig ist, 20.07.2019. https://taz.de/Wissenschaftsrat-ueber-Friedensforschung/! 5612581/. Zugegriffen: 10. März 2020.

Wissenschaftsrat. 2019. Empfehlungen zur Weiterentwicklung der Friedens- und Konfliktforschung. Drs. 7827-19. Gießen: Wissenschaftsrat. 